\title{
Effect of Compound Chuanxiong Capsule on Inflammatory Reaction and PI3K/Akt/NF- $\kappa$ B Signaling Pathway in Atherosclerosis
}

\author{
Qunfu Kang, ${ }^{1}$ Weihong Liu, ${ }^{1,2}$ Hongxu Liu, ${ }^{1}$ and Mingxue Zhou ${ }^{1,2}$ \\ ${ }^{1}$ Beijing Hospital of Traditional Chinese Medicine, Capital Medical University, Beijing 100010, China \\ ${ }^{2}$ Beijing Institute of Traditional Chinese Medicine, Beijing 100010, China \\ Correspondence should be addressed to Weihong Liu; wh.1-007@163.com and Mingxue Zhou; mingxue78@163.com
}

Received 1 April 2015; Revised 31 May 2015; Accepted 11 June 2015

Academic Editor: Zhang Tan

Copyright ( 92015 Qunfu Kang et al. This is an open access article distributed under the Creative Commons Attribution License, which permits unrestricted use, distribution, and reproduction in any medium, provided the original work is properly cited.

Compound Chuanxiong Capsule (CCC), a Chinese herbal compound, can exhibit antiatherosclerotic effect; however, its mechanism is still unclear. This study is designed to study the mechanism of CCC on atherosclerosis in the ApoE-knockout $\left(\mathrm{ApoE}^{-/-}\right)$mice fed with a high-fat diet. After 6 weeks of high-fat feeding, $40 \mathrm{ApoE}^{-/-}$mice were randomized $(n=10)$ and treated with lipitor, high-dose or low-dose CCC, or distilled water (ApoE ${ }^{-1-}$ group) for 7 weeks. The blood lipids in serum and the plaque areas of the mice were measured and the mRNA expressions of phosphatidylinositol-3-kinases (PI3K), Akt, nuclear factor-kappa B $(\mathrm{NF}-\kappa \mathrm{B})$, tumor necrosis factor- $\alpha$ (TNF- $\alpha$ ), and interleukin-6 (IL-6) of the aortae were determined. The data showed that CCC can significantly decrease the levels of blood lipids, atherosclerosis index, and plaque areas and increase collagen proportion in plaques as compared with the untreated mice $(p<0.05, p<0.01)$. In addition, CCC can significantly reduce the mRNA expressions of PI3K, Akt, NF- $\kappa$ B, IL- 6 , and TNF- $\alpha$ in the mice fed with a high-fat diet $(p<0.001)$. Thus, we concluded that CCC can inhibit inflammatory reaction in the $\mathrm{ApoE}^{-/-}$mice fed with a high-fat diet. This mechanism may be attributed to regulating PI3K/Akt/NF$\kappa \mathrm{B}$ signaling pathway.

\section{Introduction}

Atherosclerosis, a chronic inflammatory disease, plays a key role in the occurrence and development of the cardiovascular diseases, which has become as the most chronic cause of death worldwide [1]. The data from the report of Cardiovascular Disease in China 2013 showed that cardiovascular disease resulted in $41.1 \%$ deaths in the city and $38.7 \%$ in the rural areas [2]. Therefore, it is essential to prevent and treat atherosclerosis for the better prognosis of the patients with cardiovascular diseases.

In the inflammatory mechanism of initiating the occurrence of atherosclerosis, NF- $\kappa \mathrm{B}$ plays one of the most important roles as multifunctional transcription regulators. Once activated, it exerts crucial effects on inflammatory reaction, immune reaction, cell proliferation, and apoptosis by regulating gene expressions of inflammatory cytokines and chemokines, such as IL- 6 and TNF- $\alpha$ [3]. The expressions of
$\mathrm{NF}-\kappa \mathrm{B}$ and its cascade genes can be regulated by PI3K/Akt signaling pathway, which plays a vital role in the process of cell proliferation, apoptosis, and inflammatory reaction $[4,5]$.

CCC, one of the typical Chinese herbal compounds with the potential of activating blood circulation, is composed of Chuanxiong (Ligusticum) and Danggui (Angelica sinensis). Zhao and Niu showed that CCC can decrease the level of blood lipids in the patients with coronary heart disease [6] and can reduce the risk of heart failure, for example, the frequency and severity, and improve clinical symptoms of cardiac insufficiency [7]. In rat atherosclerosis model, it was found that CCC could increase the coronary blood flow, inhibit platelet adhesion and aggregation, reduce blood viscosity, and improve the state of blood stasis in blood vessels [8].

The mechanism of action of CCC, in TCM, is attributed to the potential of Chuanxiong and Danggui in activating blood circulation. Chuanxiong and Danggui have been widely used 
in the diseases with blood stasis syndrome [9]. It was reported that CCC can inhibit the aggregation of platelets and lower the viscosity of the blood [10]. However, the mechanism of action of CCC on atherosclerosis is still unclear.

Considering the role of $\mathrm{PI} 3 \mathrm{~K} / \mathrm{Akt} / \mathrm{NF}-\kappa \mathrm{B}$ signaling pathway and its downstream inflammatory cytokines in atherosclerosis, this study was designed to investigate the inflammatory mechanism of CCC on atherosclerosis in the ApoEknockout $\left(\mathrm{ApoE}^{-/-}\right)$mice fed with a high-fat diet by detecting the expressions of TNF- $\alpha$, IL- 6, PI3K, Akt, and NF- $\kappa$ B.

\section{Materials and Methods}

2.1. Animals and Treatment. Eight-week-old male $\mathrm{ApoE}^{-/-}$ mice ( $n=40,18-20 \mathrm{~g}$ ) on a C57BL/6J background and 10 C57BL mice introduced and bred by the Animal Unit of Peking University Health Science Center were used in the study. The housing and care of the animals and all the procedures were performed in accordance with the guidelines and regulations of the University of Bristol and the United Kingdom Home Office.

2.2. Ethics Statement. This study was approved by the Institutional Animal Care and Use Committee (IACUC) from Peking University Health Science Center.

2.3. Materials and Reagents. A TRIzol kit was purchased from Invitrogen Company (California, USA), PCR primers were synthesized by Sangon Biotech Co., Ltd. (Shanghai, China), and an M-MLV RT kit and a real-time (RT)-PCR kit were purchased from Takara Company (Otsu, Shiga, Japan). The blood lipid kits were purchased from Zhongsheng Beikong Biotechnology Co., Ltd. (Beijing, China) to measure total cholesterol (TC), triglycerides (TGs), low-density lipoprotein (LDL), and high-density lipoprotein (HDL-C). Van Gieson (VG) staining kit was purchased from MAIXINBIO (Fuzhou, China). CCC was purchased from Shandong Phoenix Pharmaceutical Co., Ltd., (Shandong, China; Batch number: Z20000035) and lipitor (atorvastatin) was purchased from Pfizer Pharmaceutical Co., Ltd. (Dalian, China; Batch number: H20051408).

2.4. Establishment of Atherosclerosis Model. All the ApoE ${ }^{-/-}$ mice were fed with a high-fat diet containing $21 \%$ (wt/wt) fat from lard supplemented with $0.15 \%$ (wt/wt) cholesterol [11] and obtained from Beijing Ke'ao Xieli Feed Co. Ltd. (Beijing, China) for 13 weeks. Additionally, 10 C57BL mice fed a standard chow diet containing $4 \%$ fat were used as normal control group. All the mice were inspected at least once every $24 \mathrm{~h}$.

2.5. Drug Treatment. After 6 weeks of high-fat diet, the $\mathrm{ApoE}^{-/-}$mice were randomized into $\operatorname{ApoE}^{-/-}(n=10)$, $\operatorname{ApoE}^{-/-}+$lipotor $(n=10), \mathrm{ApoE}^{-/-}+\operatorname{CCC~low}(n=10)$, and $\mathrm{ApoE}^{-/-}+\mathrm{CCC}$ high $(n=10)$ and were treated with $2.973 \mathrm{mg} / \mathrm{kg} / \mathrm{d}$ lipitor (positive-control drug) by intragastric administration, high dose of CCC (1333.48 mg/kg per day), low dose of CCC (333.37 mg/kg per day), or distilled water (control group) for an additional 7 weeks. The feeding of the mice in all the groups was accompanied by a high-fat diet. The choice of medical doses was based on the clinically relevant doses in humans (the conversion coefficient between human and mice is 9.01, and the medical doses in mice are $9.01 \times$ the clinically medical doses in humans [12]). Distilled water was used to dilute the medicine. Distilled water consumption was monitored twice weekly, and drug concentration was adjusted as required.

2.6. Histology. All the mice in the five groups were euthanized with $0.1 \%$ pentobarbital sodium. The heart from each mouse was removed and the one-third of the apical heart, including the aortic sinus, was fixed in 10\% formaldehyde, embedded, and sectioned to determine the morphology of any atherosclerotic plaque by hematoxylin and eosin (HE) and VG staining.

2.7. Evaluation of Atherosclerotic Lesions. To quantitatively evaluate atherosclerotic lesions, eight sections ( $5 \mu \mathrm{m}$-thick) per group were selected and the sections in the same segment were quantified according to Suzuki et al. [13]. Ten slides per group were examined for morphometric analysis. The average of the measured four sections per sample was recorded. The plaque morphology and the collagen content of atherosclerotic plaque were evaluated by $\mathrm{HE}$ and VG staining. A morphometric analysis was performed using Image Pro Plus (Media Cybernetics, Rockville, MD, USA). The plaque area was measured directly and was subtracted from the area enclosed by the internal elastic lamina to derive the patent lumen area [14], but the plaque area should be corrected by dividing the internal elastic lamina surrounding area.

2.8. Determination of Plasma Lipid Concentration. Blood samples were drawn from the left ventricle of a cohort of all male ApoE ${ }^{-/-}$mice that received a high-fat diet for 13 weeks. TC and TGs were determined by enzyme studies in serum. LDL-C and HDL-C levels were determined by immunoturbidimetry. Finally, all the indices were determined using the RX-2000 radiometer (Technicon Instruments Company, Tarrytown, NY, USA), and the atherosclerosis index (AI) was calculated using the formula [15]:

$$
\mathrm{AI}=\frac{\text { non-HDL-C }}{\mathrm{HDL}-\mathrm{C}}
$$

2.9. Real-Time Quantitative PCR. The aortic root from each mouse from all the groups was removed and stored in $-80^{\circ} \mathrm{C}$ to examine the mRNA expressions of PI3K, Akt, NF- $\kappa \mathrm{B}, \mathrm{IL}-6$, and TNF- $\alpha$. The total RNA from aortae was extracted using TRIzol kit according to the manufacturer's instructions. The primers of PI3K, Akt, NF- $\kappa \mathrm{B}, \mathrm{IL}-6$, and TNF- $\alpha$ are shown in Table 1. The protocol used for RT-PCR was similar to the previously described method [16]. The model of qPCR machine was ABI 7500 (America) and the program used was SDS 1.3 version. The data was analyzed by $2^{-\Delta \Delta C T}$ method, 
TABLE 1

\begin{tabular}{lll}
\hline Genes & Forward & Reverse \\
\hline GAPDH & $5^{\prime}$-GCAAGTTCAACGGCACAG-3' & $5^{\prime}$-CGCCAGTAGACTCCACGAC-3' \\
PI3K & $5^{\prime}$-TCCAAATACCAGCAGGATCA-3 & $5^{\prime}$-ATGCTTCGATAGCCGTTCTT-3 \\
Akt & $5^{\prime}$-TACTCATTCCAGACCCACGA-3' & $5^{\prime}$-GAGGTTCTCCAGCTTCAGGT-3' \\
NF- $\kappa$ B & $5^{\prime}$-GGAGAAGGCTGGAGAAGATG-3' & $5^{\prime}$-GCTCATACGGTTTCCCATTT-3' \\
IL-6 & $5^{\prime}$-GGACCAAGACCATCCAATTC-3' & $5^{\prime}$-ACCACAGTGAGGAATGTCCA-3' \\
TNF- $\alpha$ & $5^{\prime}$-CTAGCCAGGAGGGAGAACAG-3' & $5^{\prime}$-GCTTTCTGTGCTCATGGTGT-3' \\
\hline
\end{tabular}
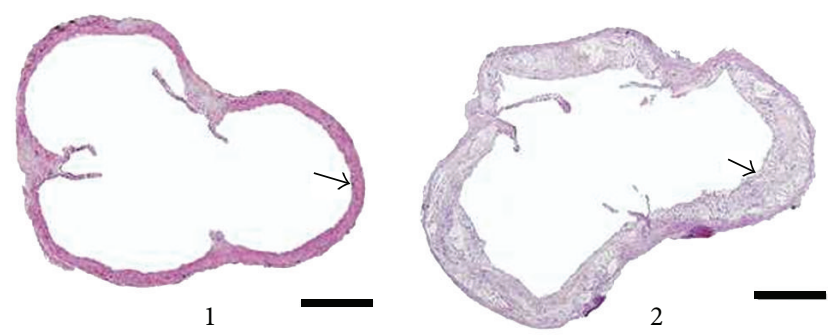

(a)
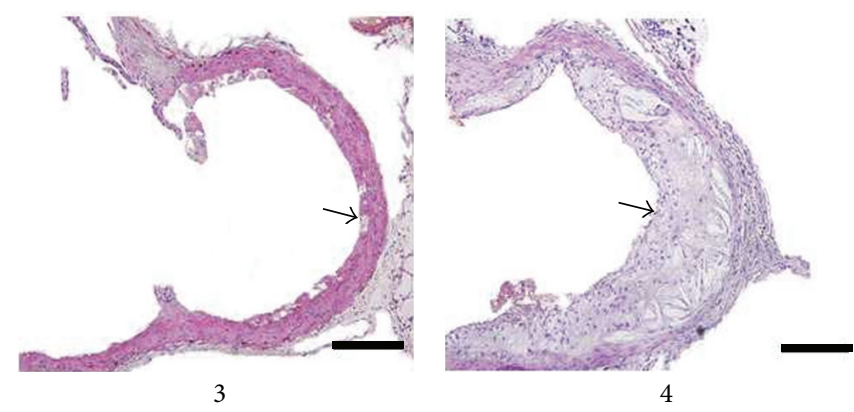

(b)

FIGURE 1: Comparison of pathological morphology of the aorta of the C57BL/6J mice and the ApoE ${ }^{-/-}$mice under different magnification at 13 weeks after being fed with different diets. (a) The pathological morphology of the aortae of the C57BL/6 $\mathrm{Jand}_{\mathrm{ApoE}}{ }^{-/-}$mice under $\times 100$ magnification at 13 weeks after being fed with different kinds of diets (scale bars $=500 \mu \mathrm{m}$ ). (b) The pathological morphology of the aortae of the $\mathrm{C} 57 \mathrm{BL} / 6 \mathrm{~J}$ and $\mathrm{ApoE} \mathrm{E}^{-/-}$mice under $\times 200$ magnification at 13 weeks after being fed with different kinds of diets (scale bars $=200 \mu \mathrm{m}$ ). Hematoxylin and eosin (HE) staining: the black arrow indicates the aorta of the mice.

and the internal reference gene used was glyceraldehyde 3phosphate dehydrogenase (GAPDH).

2.10. Statistical Analysis. Mean values and standard deviations (mean \pm S.D.) were calculated for each of the variables. All the statistical procedures were performed using Statistical Package for the Social Sciences (SPSS) 11.5. Normally distributed data was analyzed using one-way analysis of variance (ANOVA), while the statistical significance of intergroup differences in all the tested variables was evaluated using Bonferroni post hoc test. In all the cases, $p<0.05$ was as statistically significant.

\section{Results}

3.1. High-Fat Diet Induced Atherosclerosis in ApoE ${ }^{-1-}$ Mice Model. After the $\mathrm{ApoE}^{-/-}$mice were fed with high-fat diet for 13 weeks, the atherosclerotic plaques in the aortic valves attachment sites, including cholesterol crystal and foam cells, were observed in the aortic roots of the $\mathrm{ApoE}^{-1-}$ mice, whereas no plaques were observed in the aortic roots of the C57 mice (Figure 1).

3.2. Effects of CCC on Blood Lipids. The results showed that, in comparison with the $\mathrm{ApoE}^{-/-}$group mice, the levels of TGs, TC, and LDL-C in serum of the $\mathrm{ApoE}^{-/-}$mice in the high-dose and low-dose CCC groups were significantly decreased $(p<0.05)$, but the level of HDL-C in serum was not significantly altered $(p>0.05)$ (Figures $2(a)-2(d))$.

3.3. Effects of CCC on Atherosclerotic Index. The atherosclerotic indexes of mice in the $\mathrm{ApoE}^{-/-}+$lipitor group and the $\mathrm{ApoE}^{-/-}+\mathrm{CCC}$ low and $\mathrm{ApoE}^{-/-}+\mathrm{CCC}$ high groups were significantly decreased as compared with the $\mathrm{ApoE}^{-/-}$group mice $(p<0.05)$ (Figure 2(e)).

3.4. Effects of CCC on the Atherosclerotic Plaques and Its Compositions. The corrected areas of atherosclerotic plaque in the mice of the $\mathrm{ApoE}^{-/-}+$lipitor and the $\mathrm{ApoE}^{-/-}+$ CCC low groups were significantly decreased $(p<0.01)$. Moreover, the area percentage of collagen in the atherosclerotic plaques of the mice in $\mathrm{ApoE}^{-/-}+\mathrm{CCC}$ high group was significantly increased $(p<0.01)$ as compared to the mice in $\mathrm{ApoE}^{-/-}$group, while the area percentage of extracellular lipid of the two $\mathrm{ApoE}^{-/-}+\mathrm{CCC}$ groups was decreased, but not significantly changed $(p>0.05)$ (Figure 3 ).

3.5. Effects of CCC on mRNA Expressions of IL-6 and TNF- $\alpha$. As shown in Figure 4, the mRNA expression of IL-6 in mice in the $\mathrm{ApoE}^{-/-}+\mathrm{CCC}$ high group was more significantly decreased than in mice in the $\mathrm{ApoE}^{-/-}$group $(p<0.0001)$. Additionally, the mRNA expression of TNF- $\alpha$ in mice in the $\mathrm{ApoE}^{-/-}+$CCC low group was significantly decreased $(p<$ $0.0001)$. 


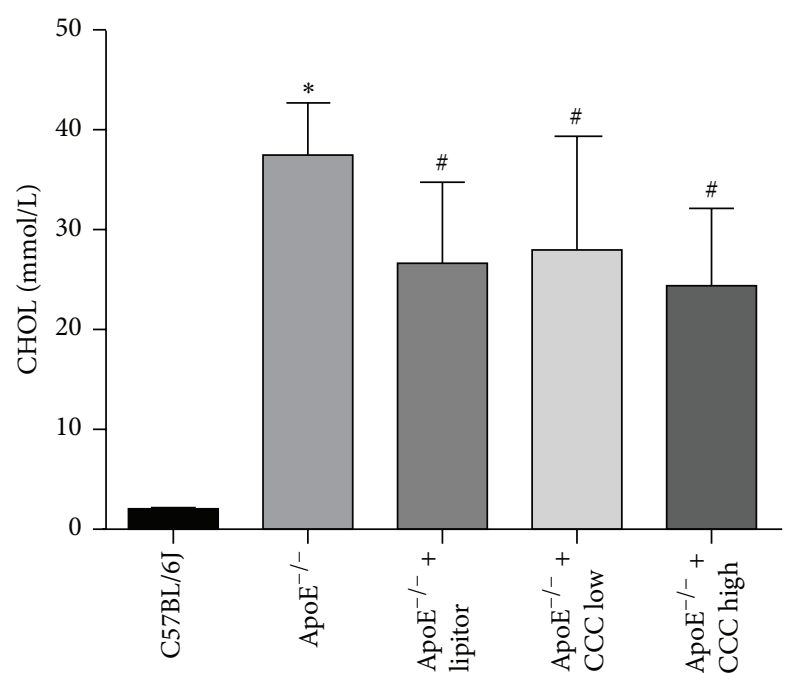

(a)

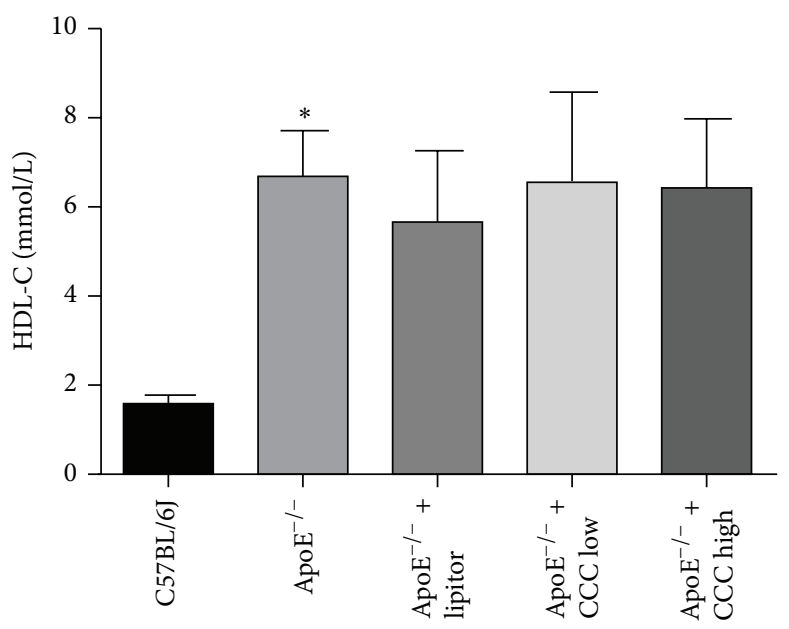

(c)

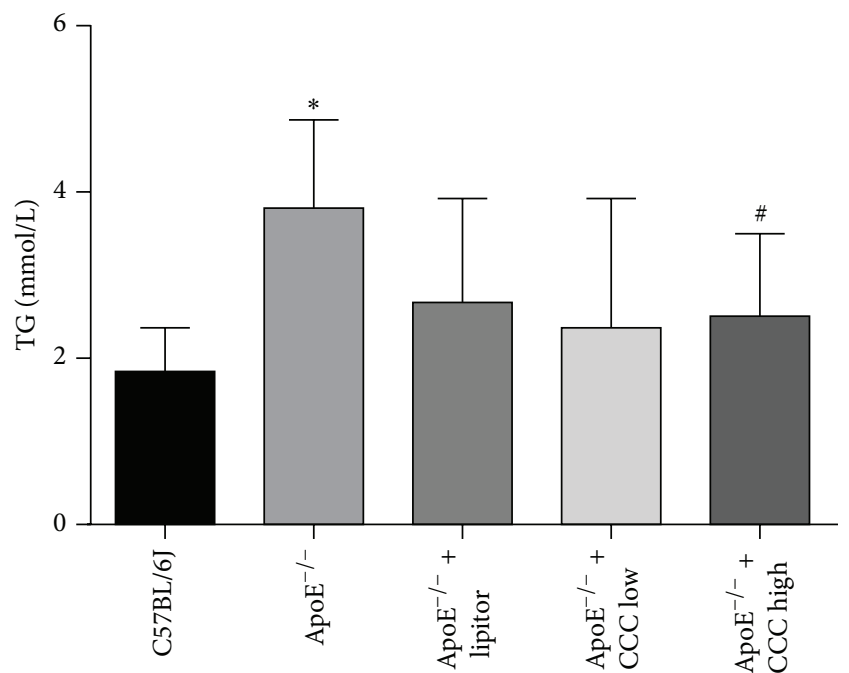

(b)

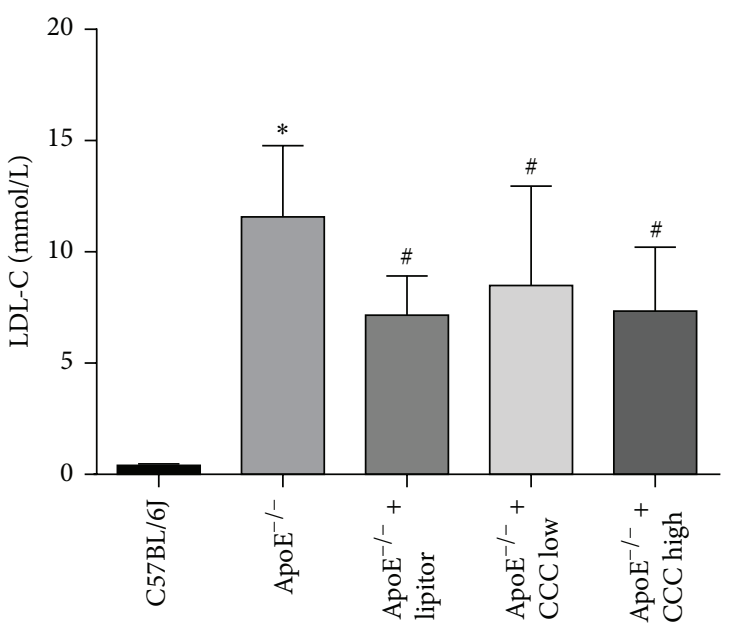

(d)

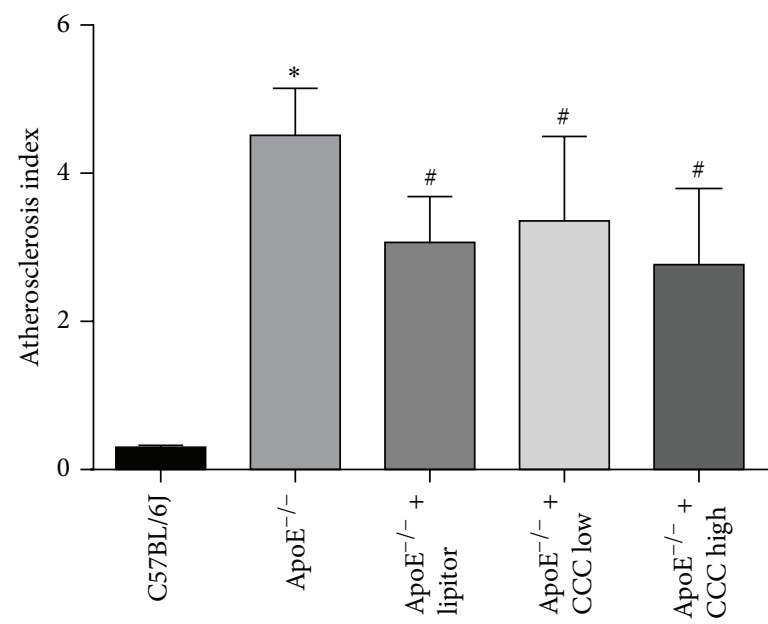

(e)

Figure 2: Effects of Compound Chuanxiong Capsule (CCC) on blood lipids in serum of ApoE ${ }^{-/-}$mice fed with high-fat diet. (a) Total cholesterol (CHOL). (b) Triglyceride (TG). (c) Low-density lipoprotein cholesterol (LDL-C). (d) High-density lipoprotein cholesterol (HDLC). (e) Atherosclerotic index (AI, AI = non-HDL-C/HDL). ${ }^{*} p<0.05$ versus C57BL/6J group; ${ }^{*} p<0.05$ versus ApoE ${ }^{-/-}$group (C57BL/6J:

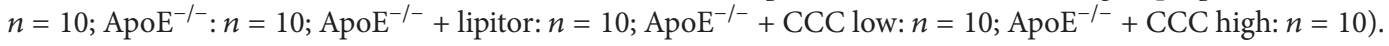




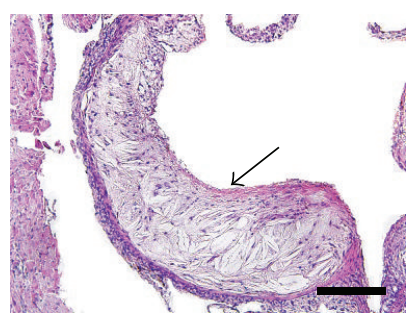

1

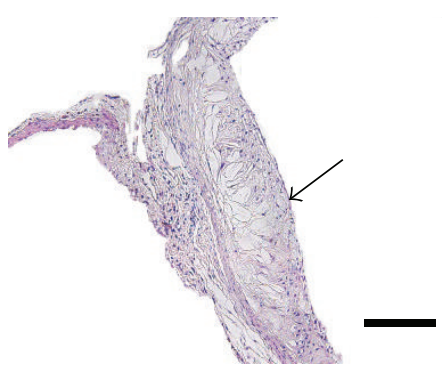

3

(a)

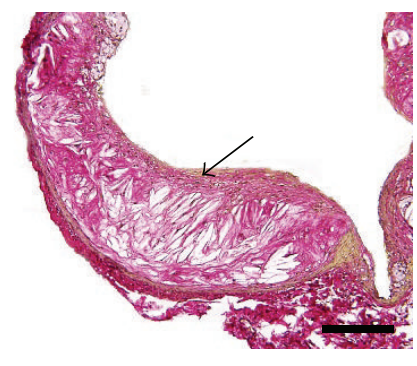

1

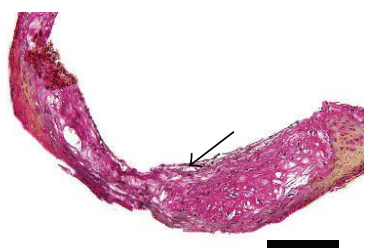

3

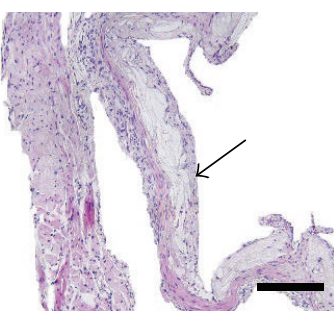

2

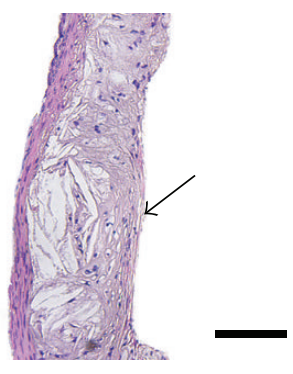

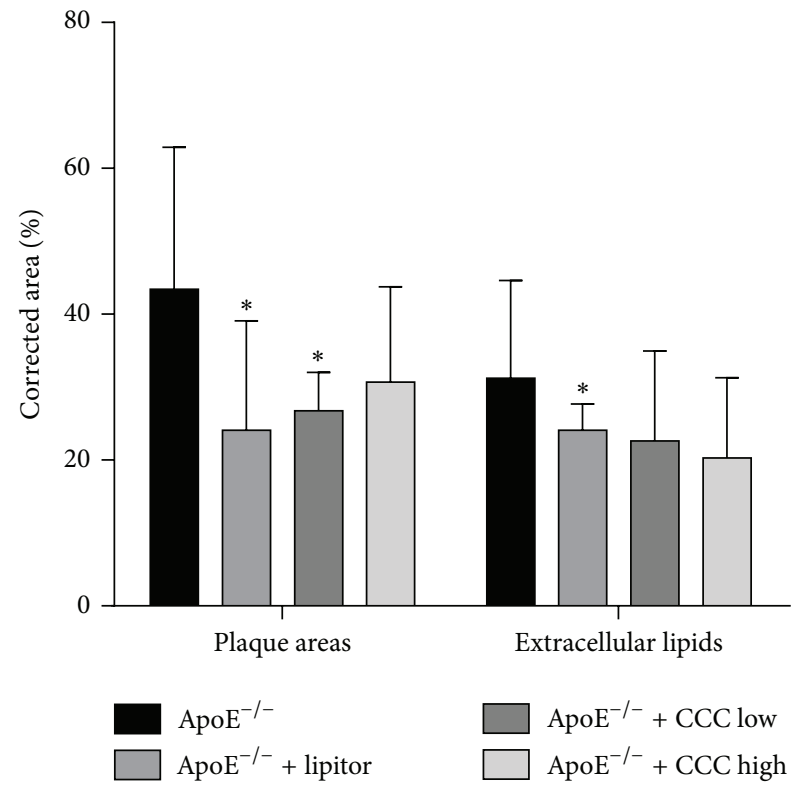

(b)

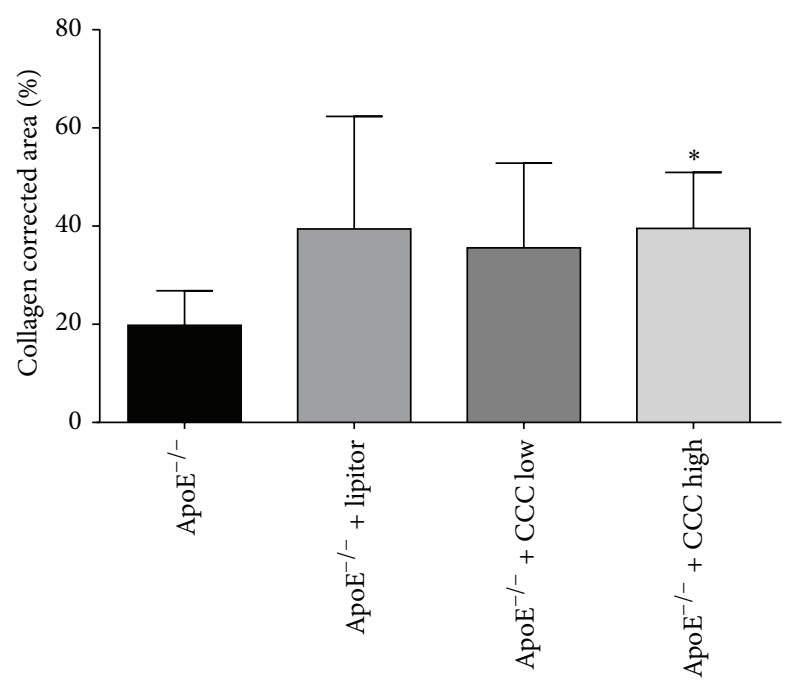

(d)

Figure 3: Effect of Compound Chuanxiong Capsule (CCC) on atherosclerotic plaque. (a) Hematoxylin and eosin (HE) staining showing the pathological morphology change of the atherosclerotic plaque in aorta of the $\mathrm{ApoE}^{-/-}$mice after the treatment of CCC. 1: ApoE ${ }^{-/-}$group; 2 : $\mathrm{ApoE}^{-/-}+$lipitor group; 3: ApoE $\mathrm{E}^{-/-}+\mathrm{CCC}$ low group; 4: $\mathrm{ApoE}^{-/-}+\mathrm{CCC}$ high group. Scale bars $=200 \mu \mathrm{m}$, and the black arrow indicates the atherosclerotic plaque in aorta. (b) The statistical analysis of the corrected area of atherosclerotic plaque and extracellular lipids content in plaque of the $\mathrm{ApoE}^{-/-}$mice after the treatment of CCC. ${ }^{*} p<0.01$ versus ApoE ${ }^{-/-}$group. (c) Van Gieson (VG) staining showing the pathological morphology change of content in atherosclerotic plaque in aorta of the Apo: ${ }^{-/-}$mice after the treatment of $\mathrm{CCC}^{1}$ : ApoE ${ }^{-/-}$ group; 2: ApoE $\mathrm{E}^{-/-}+$lipitor group; 3: $\mathrm{ApoE}^{-/-}+\mathrm{CCC}$ low group; 4: $\mathrm{ApoE}^{-/-}+\mathrm{CCC}$ high group. Scale bars $=200 \mu \mathrm{m}$, and the black arrow indicates the collagen content in plaque. (d) The statistical analysis of the corrected area of collagen in atherosclerotic plaque of the ApoE ${ }^{-/-}$ mice after the treatment of CCC. ${ }^{*} p<0.01$ versus ApoE ${ }^{-/-}$group $\left(\mathrm{ApoE}^{-/-}: n=10 ; \mathrm{ApoE}^{-/-}+\right.$lipitor: $n=10$; ApoE ${ }^{-/-}+\mathrm{CCC}$ low: $n=10$; $\mathrm{ApoE}^{-/-}+$CCC high: $\left.n=10\right)$.

3.6. Effects of CCC on mRNA Expressions of PI3K, Akt, and $N F-\kappa B$. As shown in Figure 5, the mRNA expressions of $\mathrm{PI} 3 \mathrm{~K}, \mathrm{Akt}$, and NF- $\kappa \mathrm{B}$ in mice in the $\mathrm{ApoE}^{-/-}+\mathrm{CCC}$ group were significantly decreased as compared to the mice in the ApoE $^{-/-}$group $(p<0.0001)$, while the mRNA expressions of PI3K and Akt in mice in the $\mathrm{ApoE}^{-/-}+$CCC low were decreased, but not significantly changed $(p>0.05)$.

3.7. Effects of CCC on Protein Expressions of PI3K and p-Akt. As shown in Figure 6, the protein expression of PI3K in mice 


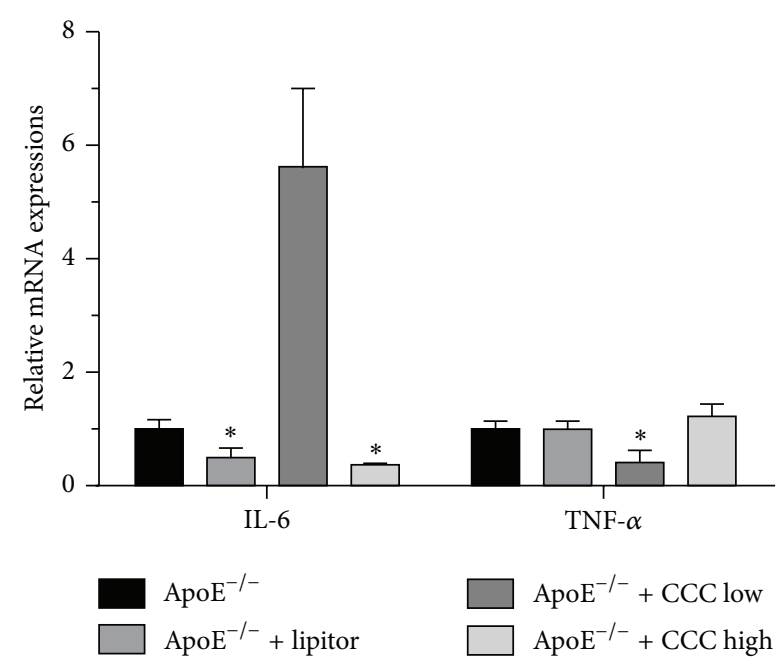

FIgURE 4: Effect of Compound Chuanxiong Capsule (CCC) on mRNA expressions of IL- 6 and TNF- $\alpha$ in $\mathrm{ApoE}^{-/-}$mice fed with high-fat diet. ${ }^{*} p<0.001$ versus $A p o E^{-/-}$group. mRNA indicates messenger RNA (ApoE ${ }^{-/-}: n=10$; ApoE ${ }^{-/-}+$lipitor: $n=10$; ApoE ${ }^{-/-}+$CCC low: $n=10 ;$ ApoE $^{-/-}+$CCC high: $\left.n=10\right)$.

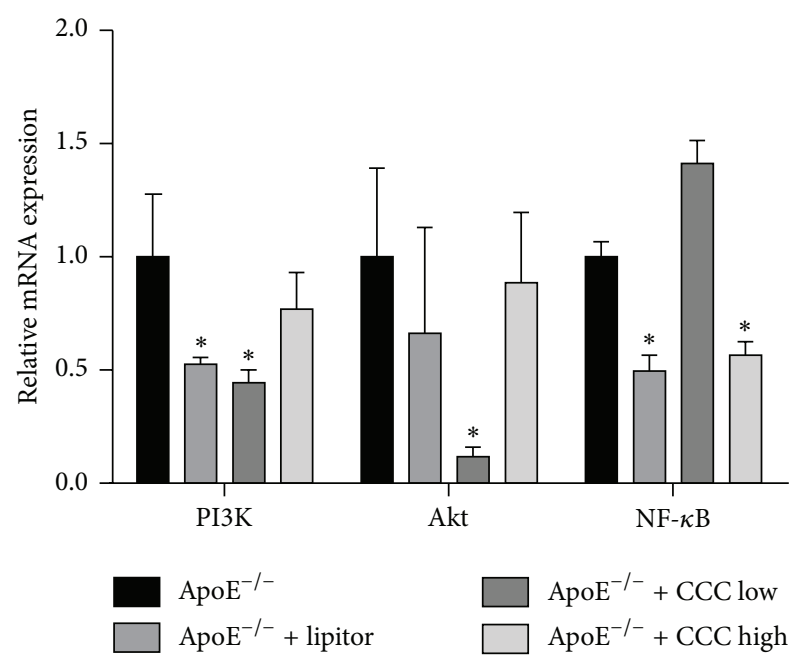

FIgure 5: Effect of Compound Chuanxiong Capsule (CCC) on mRNA expressions of PI3K, Akt and NF- $\kappa$ B. ${ }^{*} p<0.001$ versus ApoE ${ }^{-/-}$group. mRNA indicates messenger RNA $\left(\mathrm{ApoE}^{-/-}: n=10\right.$; ApoE ${ }^{-/-}+$lipitor: $n=10$; ApoE ${ }^{-1-}+$ CCC low: $n=10$; $\mathrm{ApoE}^{-/-}+$ CCC high: $n=10)$.

in the $\mathrm{ApoE}^{-/-}+\mathrm{CCC}$ low and high group was significantly decreased as compared to the mice in the $\mathrm{ApoE}^{-/-}$group $(p<0.01)$. The protein expression of $\mathrm{p}$-Akt in the $\mathrm{ApoE}^{-/-}+$ CCC low group was decreased, but not significantly changed, as compared to the mice in the $\mathrm{ApoE}^{-/-}$group $(p>0.05)$.

\section{Discussion}

In this study, we have demonstrated that CCC can dramatically ameliorate atherosclerosis in the $\mathrm{ApoE}^{-/-}$mice fed with high-fat diet by improving the blood lipid levels and reducing the plaque areas, which is consistent with the results of the previous studies $[7,10,17]$. In addition, we showed that CCC may inhibit the expression of IL- 6 and TNF- $\alpha$ by regulating $\mathrm{PI} 3 \mathrm{~K} / \mathrm{Akt} / \mathrm{NF}-\kappa \mathrm{B}$ signaling pathway in transcriptional level, which may be the main mechanism of CCC exerting the antiatherosclerotic effect.

CCC is one of the typical Chinese herbal compounds, which has been widely used in clinical practices at the physiological relevance dosage of $4 \mathrm{~T}$ and Tid $(666.7 \mathrm{mg} / \mathrm{kg}$ per day). It was shown that CCC, at the dosage of $666.7 \mathrm{mg} / \mathrm{kg}$ per day, could decrease the blood lipids and C-reactive protein significantly [18] and improves the symptoms of cardiac insufficiency $[7,19]$. In this study, the results showed that CCC inhibited atherosclerosis by regulating $\mathrm{PI} 3 \mathrm{~K} / \mathrm{Akt} / \mathrm{NF}-\kappa \mathrm{B}$ signaling pathway. The potential active ingredients of CCC, we supposed, might be ligustrazine, the main active ingredient of Chuanxiong, and ferulic acid, the main active ingredients of both Chuanxiong and Danggui. Previous studies showed that ligustrazine exhibited protective effects by reducing the levels of TNF- $\alpha$ [20] and ox-LDL [21] and others Ferulic acid could significantly decrease TC and the ratio of apo B to apo $\mathrm{A}$ and prevent the formation of aortic fatty plaques [22]. However, $\mathrm{Xu}$ et al. [23] reported that the contents of ligustrazine in the mixture of Chuanxiong and Danggui were higher than it in the Chuanxiong alone, while Danggui was devoid of ligustrazine. Thus, the inhibitory effect of CCC on atherosclerosis may not be contributed to the individual pharmacological effect of Chuanxiong or Danggui, but the overall effect of CCC, which was consistent with the holistic concept of TCM.

In the present study, the data showed that CCC can significantly decrease the blood lipid, especially TC and LDL$\mathrm{C}$, in the mice. CCC is composed of Chuanxiong (Ligusticum) and Danggui (Angelica sinensis); thus its action was attributed to the effects of ligustrazine, the main ingredients of Chuanxiong, or ferulic acid, the main ingredient of both Chuanxiong and Danggui, since the data from the previous studies showed that ligustrazine and ferulic acid can decrease blood lipids and produce a tangible protection in atherosclerosis.

The extracellular lipids and collagen in atherosclerotic plaque were responsible for its stability of atherosclerotic plaque. The data showed that CCC can decrease the percentage of extracellular lipids and increase the percentage of collagen in atherosclerotic plaque. This finding was suggestive that CCC may have the potential effect of promoting the stability of atherosclerotic plaque.

Atherosclerosis has been considered as a chronic inflammatory disease since 1999 [24] and inflammatory factors play an important role in its occurrence and development of atherosclerosis [25-27]. IL-6, an intense inflammatory cytokine, plays a critical role in cardiovascular disease [28], and it can activate endothelial cells and regulate the extracellular lipids [29]. Moreover, IL-6 can induce the synthesis of matrix metalloproteinases and regulate their function to make the atherosclerotic plaques vulnerable [30, 31]. TNF- $\alpha$ is a vital cytokine involved in the progress of atherosclerosis [32]. Kivirikko et al. showed that TNF- $\alpha$ can inhibit the expression of $\mathrm{P} 4 \mathrm{H}$, which promotes the formation of collagen 


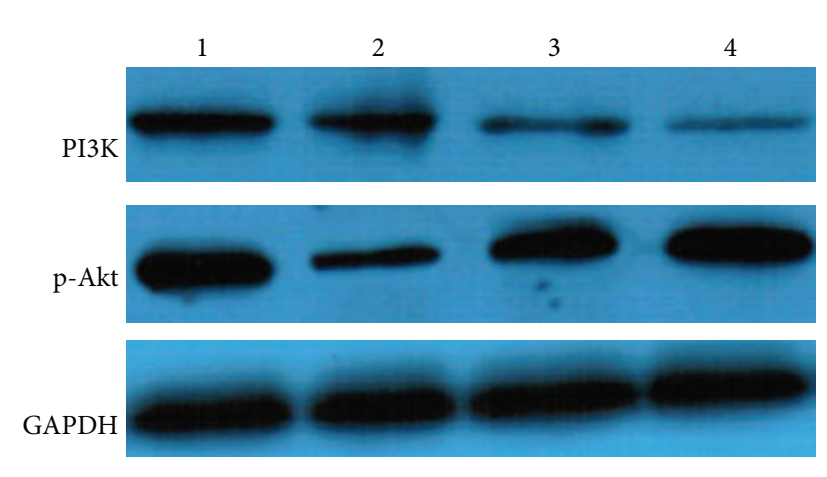

(a)

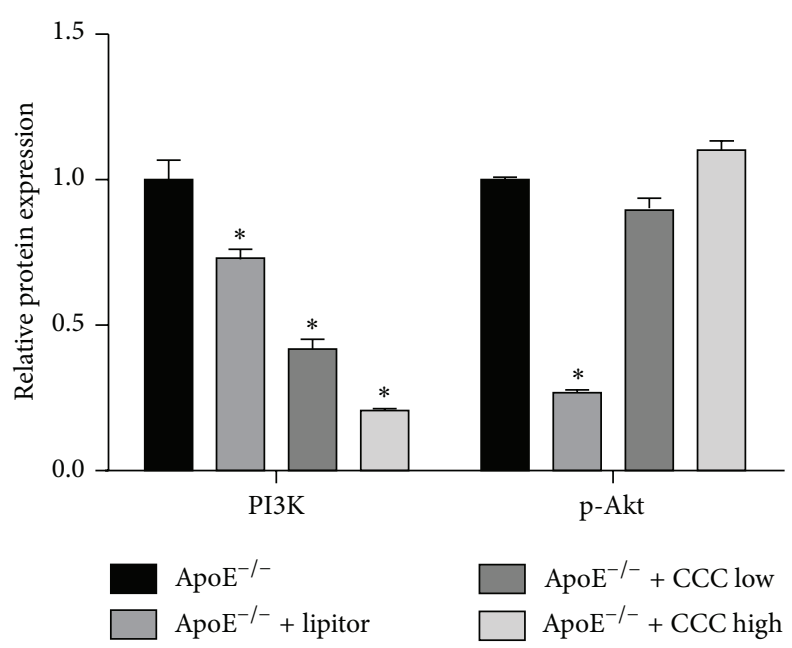

(b)

FIGURE 6: Effect of of Compound Chuanxiong Capsule (CCC) on protein expressions of PI3K and p-Akt. (a) Western blotting (WB) results of PI3K and p-Akt levels in mouse aorta. (b) Quantitative analysis (column diagram) of PI3K and p-Akt levels in mouse aorta based on WB results. ${ }^{*} p<0.05$ versus ApoE ${ }^{-/-}$group (ApoE ${ }^{-/-}: n=6$; $\mathrm{ApoE}^{-/-}+$lipitor: $n=6$; ApoE ${ }^{-/-}+\mathrm{CCC}$ low: $n=6$; ApoE ${ }^{-/-}+\mathrm{CCC}$ high: $n=6$ ).

that makes the atherosclerotic plaques vulnerable $[33,34]$, and it can inhibit the endothelial cells' function, induce lipid deposition, and promote the generation of inflammatory cytokines to aggravate atherosclerosis $[25,35]$. In this study, the data showed that CCC can inhibit the mRNA expression of IL- 6 and TNF- $\alpha$ in the aorta in the ApoE ${ }^{-/-}$mice fed with high-fat diet. Based on the effects of IL- 6 and TNF- $\alpha$ in inflammatory reaction and atherosclerosis, the role of CCC in preventing atherosclerosis may be related to inhibiting the inflammation reaction.

$\mathrm{NF}-\kappa \mathrm{B}$ is one of the crucial multifunctional transcription regulators. Once activated, it exerts effects on inflammatory reaction, immune reaction, cell proliferation, and apoptosis by regulating the gene expressions of inflammatory cytokines, and chemokines, such as IL-6 and TNF- $\alpha$ [3]. Kuang and Wang reported that, in $\mathrm{ApoE}^{-/-}$atherosclerosis mice model fed with high-fat diet, the level of NF- $\kappa$ B was significantly increased and exhibited a positive correlation with inflammation [36], and the results in our study showed that the expression of NF- $\kappa \mathrm{B}$ was significantly decreased in the $\mathrm{ApoE}^{-/-}+$ lipitor group and $\mathrm{ApoE}^{-/-}+\mathrm{CCC}$ high group as compared with the model group. In addition, several studies showed that both IL- 6 and TNF- $\alpha$ were under the influence of NF$\kappa \mathrm{B}[37-39]$ and, in turn, IL- 6 and TNF- $\alpha$ could promote the activation of NF- $\kappa \mathrm{B}$ to result in an inflammatory response [40-42]. In this study, our results showed that CCC can inhibit the expressions of NF- $\kappa \mathrm{B}$ in the aorta in mice fed with high-fat diet. These findings suggest that CCC may inhibit inflammatory reaction in atherosclerosis by regulating the expression of NF- $\kappa \mathrm{B}$.

PI3K signaling pathway and its main downstream effector protein kinase $\mathrm{B}(\mathrm{PKB} / \mathrm{Akt})$ participate in glucose metabolism, differentiation, proliferation, apoptosis, and migration of cells and inflammatory response, and NF- $\kappa \mathrm{B}$ is one-key transcription factors in regulating inflammation
[43]. PI3K/Akt signaling pathway can enable the NF- $\kappa \mathrm{B}$ transcription factors to increase the activity of inflammatory medium gene, which results in the generation of many cytokines. Luo et al. reported that the activation of PI3K/Akt signaling pathway could decrease the levels of reactive oxygen species and lipid deposits that can restrain the formation of plaques to reverse the progress of atherosclerosis [44]. However, it is well known that the activation of PI3K/Akt signaling pathway could induce the aggregation of inflammatory cells to promote the inflammation and accelerate the development of atherosclerosis $[45,46]$. Therefore, the inhibitory effect on the PI3K/Akt signaling pathway can inhibit the expression of NF- $\kappa \mathrm{B}$ to ameliorate the progress of atherosclerosis [4749]. Our results showed that both the high-dose and lowdose CCC can inhibit the mRNA expressions of PI3K and Akt, and high-dose CCC can significantly inhibit the mRNA expression of NF- $\kappa \mathrm{B}$ in aortae of the $\mathrm{ApoE}^{-/-}$mice fed with high-fat diet. Thus, CCC may suppress the mRNA expression of IL- 6 and TNF- $\alpha$ by regulating PI3K/Akt/NF- $\kappa$ B signaling pathway in transcriptional level. The results also showed that CCC can significantly inhibit the protein expression of PI3K, but not significantly inhibit the protein expression of p-Akt in aortae of the $\mathrm{ApoE}^{-/-}$mice fed with high-fat diet. The possible explanation is that the dose of CCC did not exert the corresponding effect. Therefore, further investigation is required to explain the possible mechanism of CCC on $\mathrm{PI} 3 \mathrm{~K} / \mathrm{Akt} / \mathrm{NF}-\kappa \mathrm{B}$ signaling pathway more explicitly.

\section{Conclusion}

The results from the study showed that CCC can dramatically ameliorate atherosclerosis in the $\mathrm{ApoE}^{-/-}$mice fed with high-fat diet. The possible mechanism was attributed to the inhibition of the expression of IL- 6 and TNF- $\alpha$ by regulating $\mathrm{PI} 3 \mathrm{~K} / \mathrm{Akt} / \mathrm{NF}-\kappa \mathrm{B}$ signaling pathway. Therefore, it is necessary 
to further investigate the potential effect and mechanism of CCC preventing the occurrence of atherosclerosis.

\section{Conflict of Interests}

The authors declare that there is no conflict of interests regarding the publication of this paper.

\section{Acknowledgments}

This work was supported by Grants from the National Natural Science Foundation of China (no. 81303086), Beijing Natural Science Foundation (no. 7133233), Beijing Nova Program (no. Z131107000413026), and Beijing Health System-High Level Health Technology Talent Cultivation Plan (2014-3-100).

\section{References}

[1] M. Lehrke and C. Lebherz, "AAV-mediated gene therapy for atherosclerosis," Current Atherosclerosis Reports, vol. 16, no. 9, p. 434, 2014.

[2] W.-W. Chen, R.-L. Gao, L.-S. Liu et al., "China cardiovascular summary report 2013," Chinese Circulation Journal, vol. 7, pp. 487-491, 2014.

[3] M. S. Hayden and S. Ghosh, "Shared principles in NF- $\kappa$ B signaling," Cell, vol. 132, no. 3, pp. 344-362, 2008.

[4] S. Liu, H. Shen, M. Xu et al., "FRP inhibits ox-LDL-induced endothelial cell apoptosis through an Akt-NF- $\kappa$ B-Bcl-2 pathway and inhibits endothelial cell apoptosis in an apoE-knockout mouse model," The American Journal of Physiology-Endocrinology and Metabolism, vol. 299, no. 3, pp. E351-E363, 2010.

[5] J. Sun and S.-Q. Huang, "The effect of NF- $\kappa$ B signaling pathway in the formation of atherosclerosis," Modern Journal of Integrated of Traditional Chinese and Western Medicine, vol. 16, no. 15, pp. 2158-2162, 2007.

[6] Z.-B. Zhao and Y.-Y. Niu, "The effect of compound Chuanxiong capsules on the blood lipids of CHD patients," Medical Aesthetics and Cosmetology, no. 2, p. 222, 2015.

[7] H. Li, "Compound Chuanxiong Capsule treating coronary heart disease in 50 cases with mild heart failure," Shanxi Journal of Traditional Chinese Medicine, vol. 34, no. 6, pp. 647-648, 2013.

[8] Y. Huo, T.-M. Yao, Z. Liang, J. Li, Y. You, and Y.-L. Han, "Effects of Guanxin Shutong capsule on lipid metabolism and hemorrheology in rat model with experimental atherosclerosis," Journal of Liaoning University of Traditional Chinese Medicine, vol. 13, no. 11, pp. 248-250, 2013.

[9] S.-F. Huang, R.-Y. Hou, S.-S. Gao, and T.-F. Du, "Study about the frequency of the Chinese medicine used in coronary heart disease," Traditional Chinese Drug Research \& Clinical Pharmacology, vol. 20, no. 4, pp. 395-396, 2009.

[10] S.-B. Sun, Z. Lei, H.-J. Jiang, K. Fang, and F. Chen, "The research of active blood of compound Chuanxiong capsules," Herald of Medicine, vol. 28, no. 2, pp. 189-190, 2009.

[11] J. Johnson, K. Carson, H. Williams et al., "Plaque rupture after short periods of fat feeding in the apolipoprotein E-knockout mouse: model characterization and effects of pravastatin treatment," Circulation, vol. 111, no. 11, pp. 1422-1430, 2005.

[12] M.-X. Zhou, H. Xu, L. Pan, J.-Y. Wen, W.-Q. Liao, and K.-J. Chen, "Rosiglitazone promotes atherosclerotic plaque stability in fat-fed ApoE-knockout mice," European Journal of Pharmacology, vol. 590, no. 1-3, pp. 297-302, 2008.
[13] H. Suzuki, Y. Kurihara, M. Takeya et al., "A role for macrophage scavenger receptors in atherosclerosis and susceptibility to infection," Nature, vol. 386, no. 6622, pp. 292-296, 1997.

[14] J. L. Johnson, R. Fritsche-Danielson, M. Behrendt et al., "Effect of broad-spectrum matrix metalloproteinase inhibition on atherosclerotic plaque stability," Cardiovascular Research, vol. 71, no. 3, pp. 586-595, 2006.

[15] S.-K. Yan, "Several important calculated parameters of blood lipids and its clinical significance," in Proceedings of the 4th National Symposium about Lipid Analysis and 9th National Clinical Lipoprotein Conference Papers Series, vol. 4, 2008.

[16] S.-L. Qi, Y.-F. Wang, M. X. Zhou et al., "A mitochondrialocalized glutamic acid-rich protein (MGARP/OSAP) is highly expressed in retina that exhibits a large area of intrinsic disorder," Molecular Biology Reports, vol. 38, no. 5, pp. 28692877, 2011.

[17] D.-Z. Shi, X.-C. Ma, and X.-A. Gao, "The overview of blood circulation herbs on the prevention of atherosclerosis," Journal of Traditional Chinese Medicine, vol. 36, no. 7, pp. 433-435, 1995.

[18] Y. Hu, "Clinical observation of compound Chuanxiong capsule in the treatment of carotid atherosclerotic vertigo," Practical Clinical Journal of Integrated Traditional Chinese and Western Medicine, vol. 11, no. 1, pp. 8-23, 2011.

[19] Q.-T. Feng and X.-J. Qian, "The curative effect of compound Chuanxiong combined with bisoprolol treatment of right heart function insufficiency observation," Modern Diagnosis and Treatment, no. 18, pp. 4174-4175, 2014.

[20] H.-J. Wu, J. Hao, S.-Q. Wang, B.-L. Jin, and X.-B. Chen, "Protective effects of ligustrazine on TNF- $\alpha$-induced endothelial dysfunction," European Journal of Pharmacology, vol. 674, no. 2-3, pp. 365-369, 2012.

[21] G.-F. Wang, C.-G. Shi, M.-Z. Sun et al., “Tetramethylpyrazine attenuates atherosclerosis development and protects endothelial cells from ox-LDL," Cardiovascular Drugs and Therapy, vol. 27, no. 3, pp. 199-210, 2013.

[22] E. Y. Kwon, G. M. Do, Y. Y. Cho, Y. B. Park, S. M. Jeon, and M. S. Choi, "Anti-atherogenic property of ferulic acid in apolipoprotein E-deficient mice fed Western diet: comparison with clofibrate," Food and Chemical Toxicology, vol. 48, no. 8-9, pp. 2298-2303, 2010.

[23] C.-J. Xu, Y. Kong, G.-Y. Zheng, and J.-Z. Shentu, "Content of tetramethylpyrazine in the decoction of Chuanxiong rhizome and that of Chuanxiong rhizome together with Chinese angelica root," Herald of Medicine, vol. 26, no. 10, pp. 1207-1211, 2007.

[24] R. Ross, "Atherosclerosis-an inflammatory disease," The New England Journal of Medicine, vol. 340, no. 2, pp. 115-126, 1999.

[25] R. Kleemann, S. Zadelaar, and T. Kooistra, "Cytokines and atherosclerosis: a comprehensive review of studies in mice," Cardiovascular Research, vol. 79, no. 3, pp. 360-376, 2008.

[26] E. L. Gautier, T. Huby, J. L. Witztum et al., "Macrophage apoptosis exerts divergent effects on atherogenesis as a function of lesion stage," Circulation, vol. 119, no. 13, pp. 1795-1804, 2009.

[27] P. Libby, P. M. Ridker, and A. Maseri, "Inflammation and atherosclerosis," Circulation, vol. 105, no. 9, pp. 1135-1143, 2002.

[28] P. Libby, "Role of inflammation in atherosclerosis associated with rheumatoidarthritis," The American Journal of Medicine, vol. 121, supplement 1, pp. S21-S31, 2008.

[29] M. Hibi, K. Nakajima, and T. Hirano, "IL-6 cytokine family and signal transduction: a model of the cytokine system," Journal of Molecular Medicine, vol. 74, no. 1, pp. 1-12, 1996. 
[30] K. P. Sundararaj, D. J. Samuvel, Y. Li, J. J. Sanders, M. F. LopesVirella, and Y. Huang, "Interleukin-6 released from fibroblasts is essential for up-regulation of matrix metalloproteinase-1 expression by U937 macrophages in coculture: cross-talking between fibroblasts and U937 macrophages exposed to high glucose," The Journal of Biological Chemistry, vol. 284, no. 20, pp. 13714-13724, 2009.

[31] L. R. Luckett and R. M. Gallucci, "Interleukin-6 (IL-6) modulates migration and matrix metalloproteinase function in dermal fibroblasts from IL-6KO mice," British Journal of Dermatology, vol. 156, no. 6, pp. 1163-1171, 2007.

[32] A. Ozeren, M. Aydin, M. Tokac et al., "Levels of serum IL-1 $\beta$, IL-2, IL- 8 and tumor necrosis factor- $\alpha$ in patients with unstable angina pectoris," Mediators of Inflammation, vol. 12, no. 6, pp. 361-365, 2003.

[33] K. I. Kivirikko, T. Helaakoski, K. Tasanen et al., "Molecular biology of prolyl 4-hydroxylase," Annals of the New York Academy of Sciences, vol. 580, pp. 132-142, 1990.

[34] R. Nissi, H. Autio-Harmainen, P. Marttila, R. Sormunen, and K. I. Kivirikko, "Prolyl 4-hydroxylase isoenzymes I and II have different expression patterns in several human tissues," The Journal of Histochemistry and Cytochemistry, vol. 49, no. 9, pp. 1143-1153, 2001.

[35] H. Zhang, Y. Park, J. Wu et al., "Role of TNF- $\alpha$ in vascular dysfunction," Clinical Science, vol. 116, no. 3, pp. 219-230, 2009.

[36] J. Kuang and W. Wang, "Effects of nuciferine on vascular inflammation and matrix metalloproteinases in mouse with atherosclerosis," Journal of Clinical Cardiology, vol. 35, no. 1, pp. 97-100, 2015.

[37] A. R. Brasier, "The nuclear factor-kappaB-interleukin-6 signalling pathway mediating vascular inflammation," Cardiovascular Research, vol. 86, no. 2, pp. 211-218, 2010.

[38] S. Fujioka, J. Niu, C. Schmidt et al., "NF- $\kappa$ B and AP-1 connection: Mechanism of NF- $\kappa \mathrm{B}$-dependent regulation of AP-1 activity," Molecular and Cellular Biology, vol. 24, no. 17, pp. 78067819, 2004.

[39] Y.-H. Son, Y.-T. Jeong, K.-A. Lee et al., "Roles of MAPK and NF$\kappa \mathrm{B}$ in interleukin- 6 induction by lipopolysaccharide in vascular smooth muscle cells," Journal of Cardiovascular Pharmacology, vol. 51, no. 1, pp. 71-77, 2008.

[40] F. Chen and X. Shi, "NF- $\kappa$ B, a pivotal transcription factor in silica-induced diseases," Molecular and Cellular Biochemistry, vol. 234-235, pp. 169-176, 2002.

[41] P. Storz and A. Toker, "NF-kappaB signaling: an alternate pathway for oxidative stress responses," Cell Cycle, vol. 2, no. 1, pp. 9-10, 2003.

[42] K. E. de Visser, A. Eichten, and L. M. Coussens, "Paradoxical roles of the immune system during cancer development," Nature Reviews Cancer, vol. 6, no. 1, pp. 24-37, 2006.

[43] Y. Hattori, S. Hattori, and K. Kasai, "Lipopolysaccharide activates Akt in vascular smooth muscle cells resulting in induction of inducible nitric oxide synthase through nuclear factor-kappa B activation," European Journal of Pharmacology, vol. 481, no. 2-3, pp. 153-158, 2003.

[44] Y. Luo, G.-B. Sun, M. Wang et al., "Isorhamnetin inhibit macrophage apoptosis induced atherosclerosis by activating $\mathrm{PI} 3 \mathrm{~K} / \mathrm{AKT}$ and induce the production of HO-1," in Proceedings of the 1st Session of the Conference of Chinese Medicine Information, vol. 8, 2014.

[45] T. J. Cremer, P. Shah, E. Cormet-Boyaka, M. A. Valvano, J. P. Butchar, and S. Tridandapani, "Akt-mediated proinflammatory response of mononuclear phagocytes infected with Burkholderia cenocepacia occurs by a novel GSK $3 \beta$-dependent, I $\kappa \mathrm{B}$ kinase-independent mechanism," The Journal of Immunology, vol. 187, no. 2, pp. 635-643, 2011.

[46] F. Morello, A. Perino, and E. Hirsch, "Phosphoinositide 3-kinase signalling in the vascular system," Cardiovascular Research, vol. 82, no. 2, pp. 261-271, 2009.

[47] P. M. Mourani, P. J. Garl, J. M. Wenzlau, T. C. Carpenter, K. R. Stenmark, and M. C. M. Weiser-Evans, "Unique, highly proliferative growth phenotype expressed by embryonic and neointimal smooth muscle cells is driven by constitutive Akt, mTOR, and p70S6K signaling and is actively repressed by PTEN," Circulation, vol. 109, no. 10, pp. 1299-1306, 2004.

[48] J. E. Sousa, M. A. Costa, A. Abizaid et al., "Lack of neointimal proliferation after implantation of sirolimus-coated stents in human coronary arteries: a quantitative coronary angiography and three-dimensional intravascular ultrasound study," Circulation, vol. 103, no. 2, pp. 192-195, 2001.

[49] M. Poon, J. J. Badimon, and V. Fuster, "Overcoming restenosis with sirolimus: from alphabet soup to clinical reality," The Lancet, vol. 359, no. 9306, pp. 619-622, 2002. 


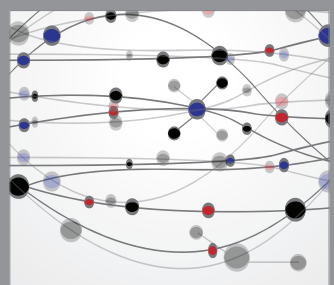

The Scientific World Journal
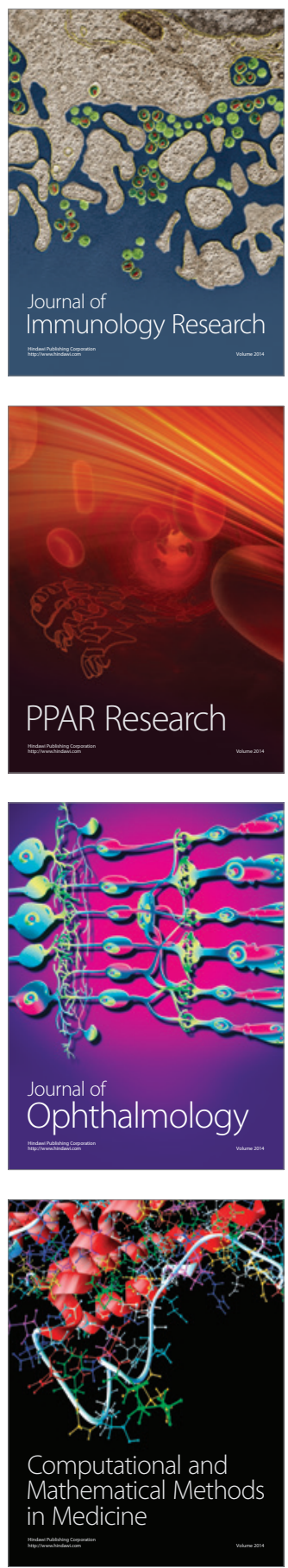

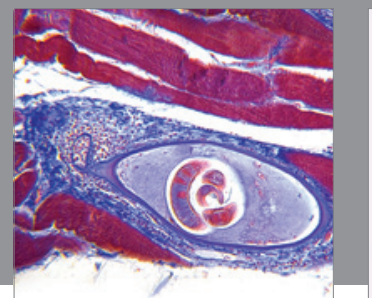

Gastroenterology

Research and Practice
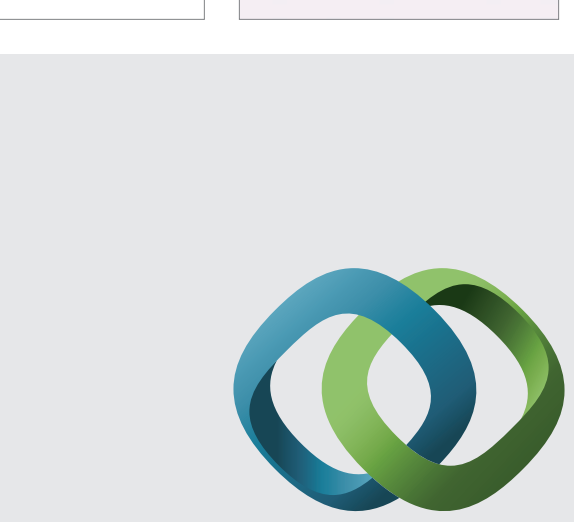

\section{Hindawi}

Submit your manuscripts at

http://www.hindawi.com
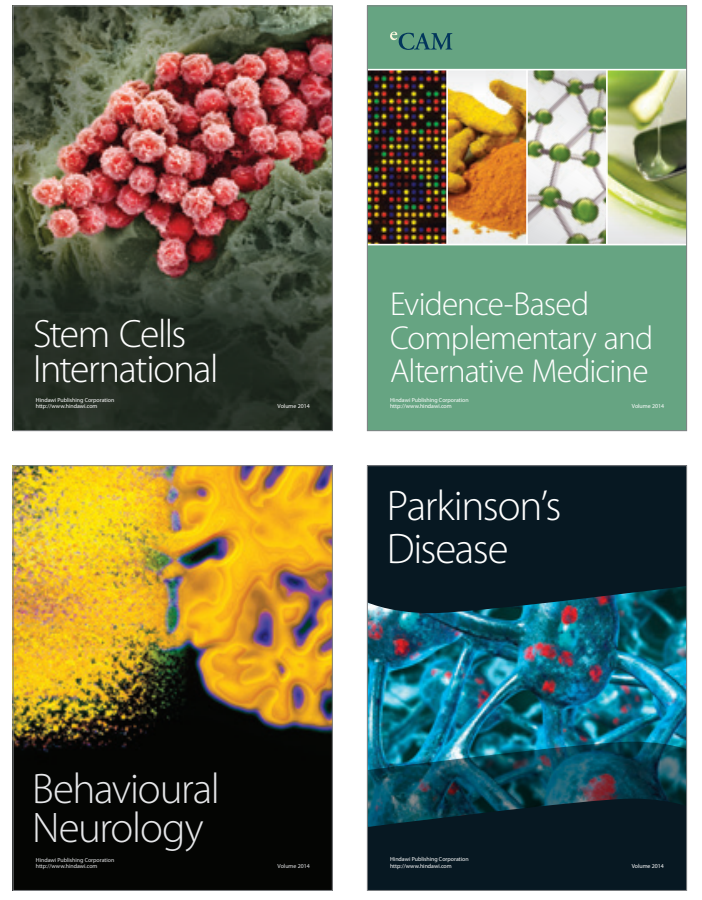
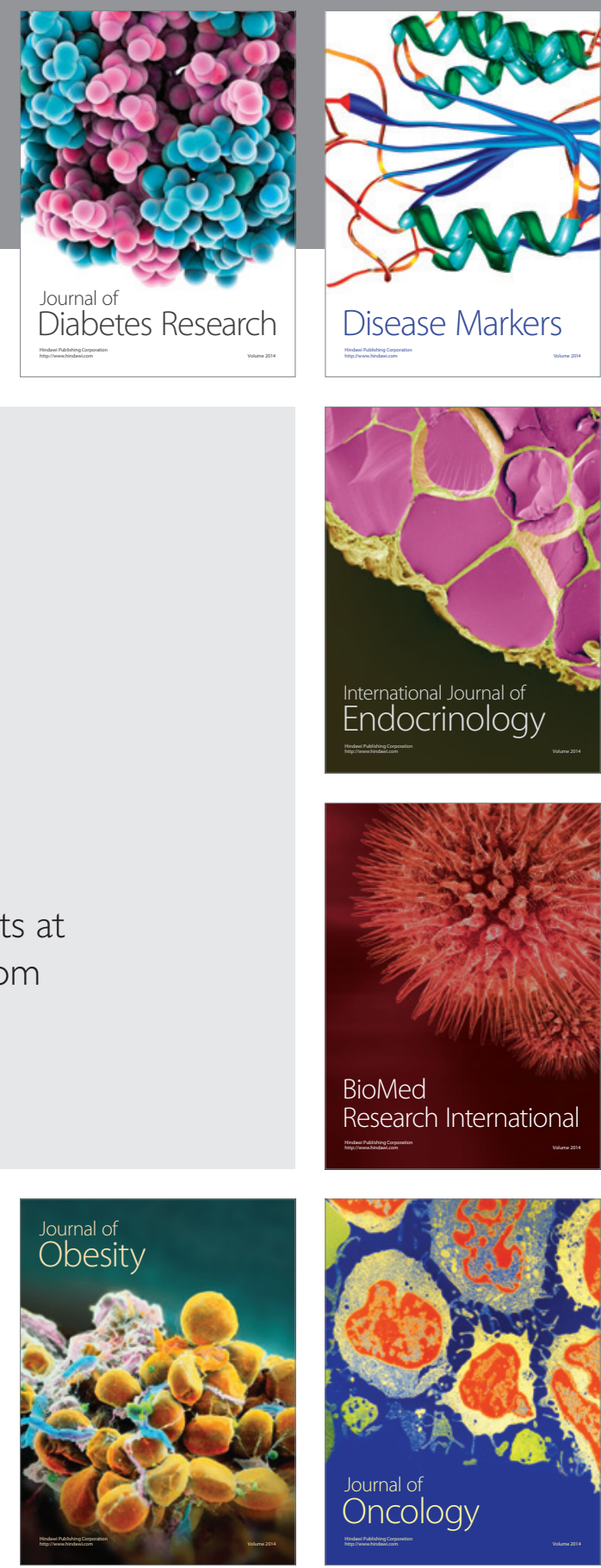

Disease Markers
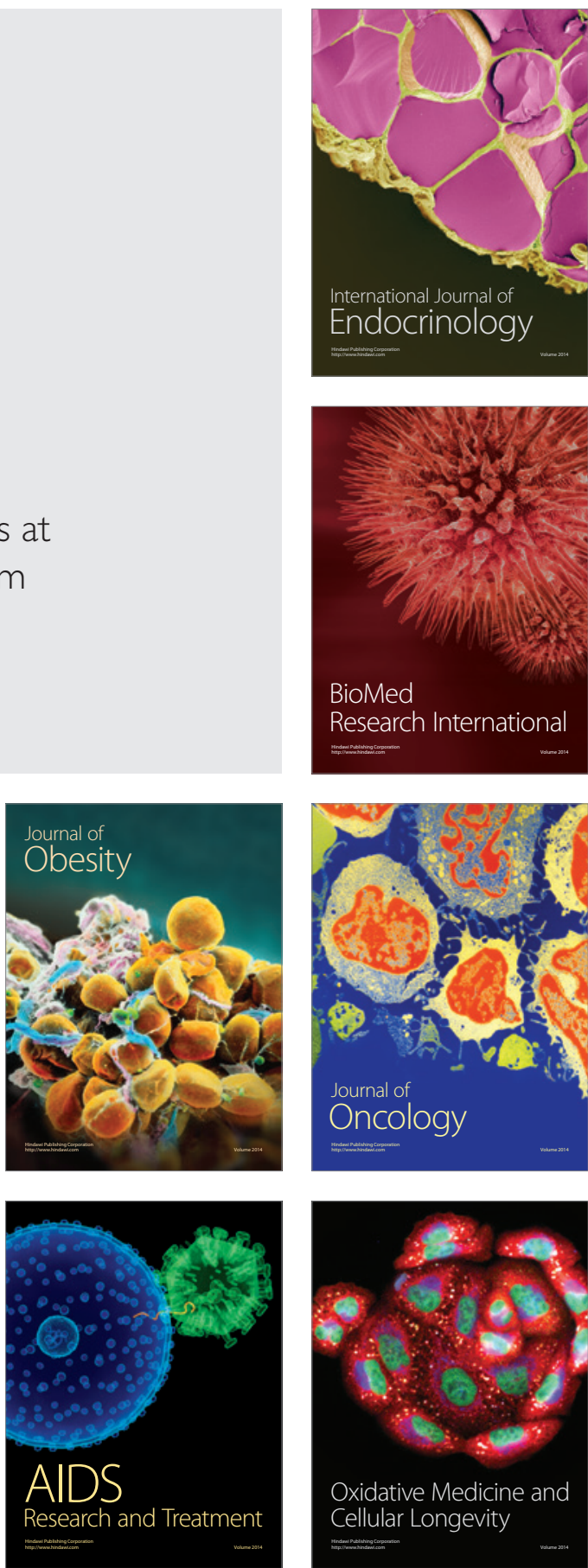